\title{
Experimental Results of High-Resolution ISAR Imaging of Ground-Moving Vehicles with a Stationary FMCW Radar
}

\author{
Janusz S. Kulpa, Mateusz Malanowski, Damian Gromek, Piotr Samczyński, Krzysztof Kulpa, and Artur Gromek
}

\begin{abstract}
In the paper experimental results of ISAR (Inverse Synthetic Aperture Radar) processing obtained with highresolution radar are presented. Targets under observation were ground moving vehicles, such as cars, trucks and tractors. The experiments were performed with a FMCW (FrequencyModulated Continuous-Wave) radar operating at $94 \mathrm{GHz}$ with almost $1 \mathrm{GHz}$ of bandwidth. Due to the measurement scenario more typical for SAR (Synthetic Aperture Radar), than ISAR, i.e. targets moving along straight line crossing the antenna beam, algorithms usually applied for SAR processing have been used.
\end{abstract}

Keywords-SAR, ISAR, FMCW, radar imaging

\section{INTRODUCTION}

$\mathbf{T}$ HE capabilities of radar have been largely extended since first devices were constructed in the first half of the 20th century. Modern radars are capable of not only detecting targets, and estimating their motion parameters - target classification and identification is also feasible. This is possible thanks to the techniques such as (among others): Synthetic Aperture Radar (SAR)/Inverse Synthetic Aperture Radar (ISAR) [1]. They are based on relative motion between the radar and the target. In SAR, which is used for imaging stationary targets and ground surface, the radar platform is moving. In ISAR, on the other hand, a stationary radar observes moving targets. The relative motion provides information on target shape, which can be extracted using advanced signal processing. As a result, a radar image of the target is obtained. Such an image may be used for target recognition or classification.

In this paper imaging of ground moving target with a stationary radar is considered [2]. The focus is on the signal processing algorithms applied in order to obtain ISAR images. For the purpose of this paper an analog front-end and analogto-digital converter module, originally designed for short-range SAR radar [3]-[5], were used. The radar front-end operates at $94 \mathrm{GHz}$ with almost $1 \mathrm{GHz}$ of bandwidth, providing very high range resolution. The radar operates in FMCW (FrequencyModulated Continuous-Wave) mode.

\section{SAR/ISAR IMAGING}

In a classical idealistic ISAR scenario, a stationary radar observes a target which is rotating in the antenna beam (see Fig. 1). As different scattering points of the target have different Doppler velocities, due to the rotation of the target,

The authors are with Warsaw University of Technology, Institute of Electronic Systems, Nowowiejska 15/19, 00-665 Warsaw, Poland (e-mail: J.Kulpa@stud.elka.pw.edu.pl). spectral analysis of the signal can provide information on the target shape. In practice simple Fast Fourier Transform (FFT) of the signal is performed to obtain ISAR image. Translational motion of the target is not necessary for ISAR imaging, as it introduces the same Doppler shift for all scatterers. Moreover, it causes additional problems during processing, as range cell migration can appear.

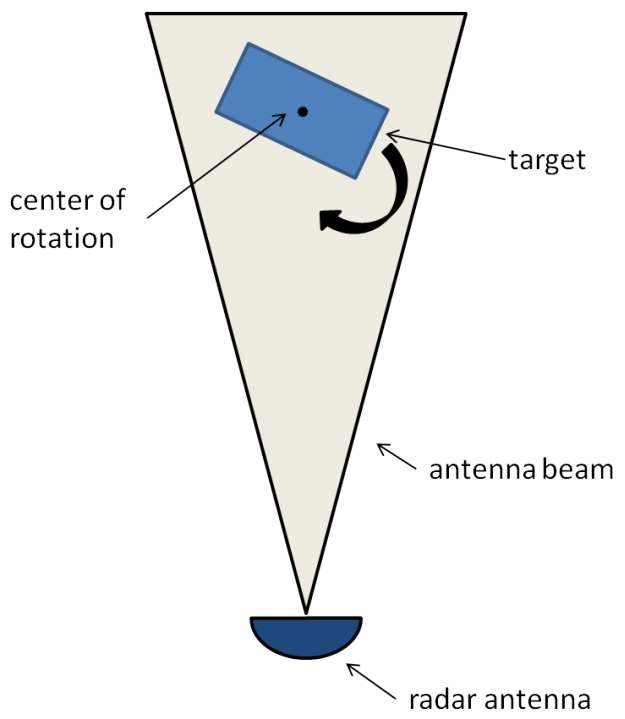

Fig. 1. Typical scenario for ISAR imaging.

The setup analyzed in this paper is substantially different from the ideal ISAR scenario (see Fig. 2). The antenna beam is pointed to the area of interest - a road. The targets are moving along the road, crossing the antenna beam. The rotation of the target within the beam is very small, however, targets show significant translational motion - the road is squinted with respect to the line of sight. As a result, strong range cell migration effect occurs.

The aforementioned real scenario differs from the ideal ISAR setup. However, there are some similarities with the squinted SAR mode. In the squinted SAR mode, the radar is placed on a moving platform, and the antenna beam is steered off the broadside. As radar platform moves, the radar - target distance differs in time. Relative velocity, which also adds up with the squint, causes the range cell migration. This situation, when the stationarity and motion of the radar and target is exchanged, is very similar to the observation of ground moving targets crossing the radar beam. Therefore, processing known 


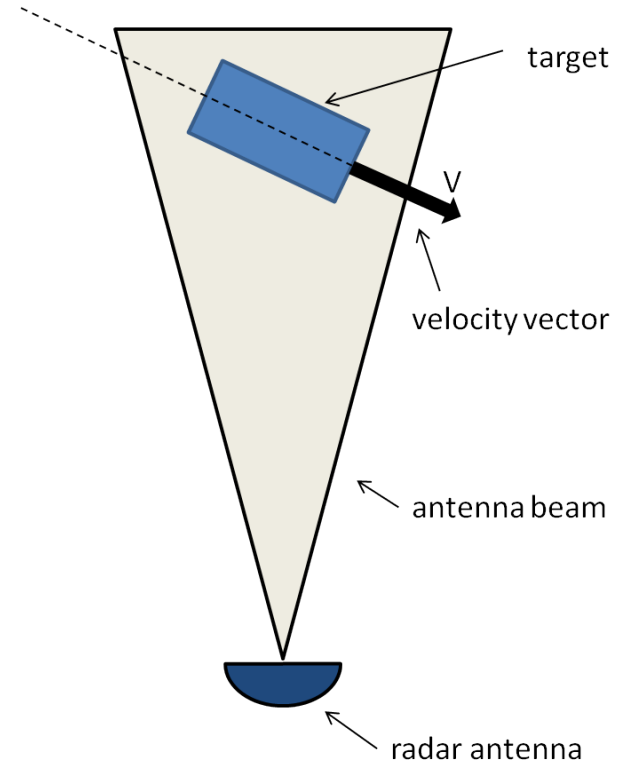

Fig. 2. ISAR imaging scenario considered in the paper.

from SAR (rather than ISAR) will be used to obtain radar imagery in this paper.

\section{SignAL PROCESSING}

The signal processing applied to obtain ISAR imagery is composed of the following steps:

\section{A. Range Compression}

The analog front-end used in the experiment operated in FMCW mode. The radar operating in this mode sends a continuous wave with frequency modulated with a sawtooth signal. At reception the received signal is mixed with the transmitted one, producing the so-called beat signal signal with differential frequency. As further targets produce echo which is delayed more, the differential frequency will be also higher. Therefore, performing spectral analysis of the beat signal, the range profile can be obtained. This process is usually called FMCW range compression, as an analogy to range compression performed in pulsed radars. In Fig. 3 the concept of the FMCW range compression is shown. The beat signal corresponding to a single scatterer at a certain distance has a form of a sine wave. The Fourier analysis, performed in practice with the FFT, results in the signal spectrum with a single peak. The frequency axis can be expressed in range to the target.

\section{B. Range Cell Migration Correction}

As it has already been indicated, in the analyzed scenario the targets are crossing the antenna beam in such a way that strong range cell migration is observed. It appears in the data as target echo moving across the range cells in consecutive pulses. This effect can be eliminated by shifting the echoes in consecutive pulses, so that the echo from a single scatterer occupies the same range resolution cell through the whole observation time.
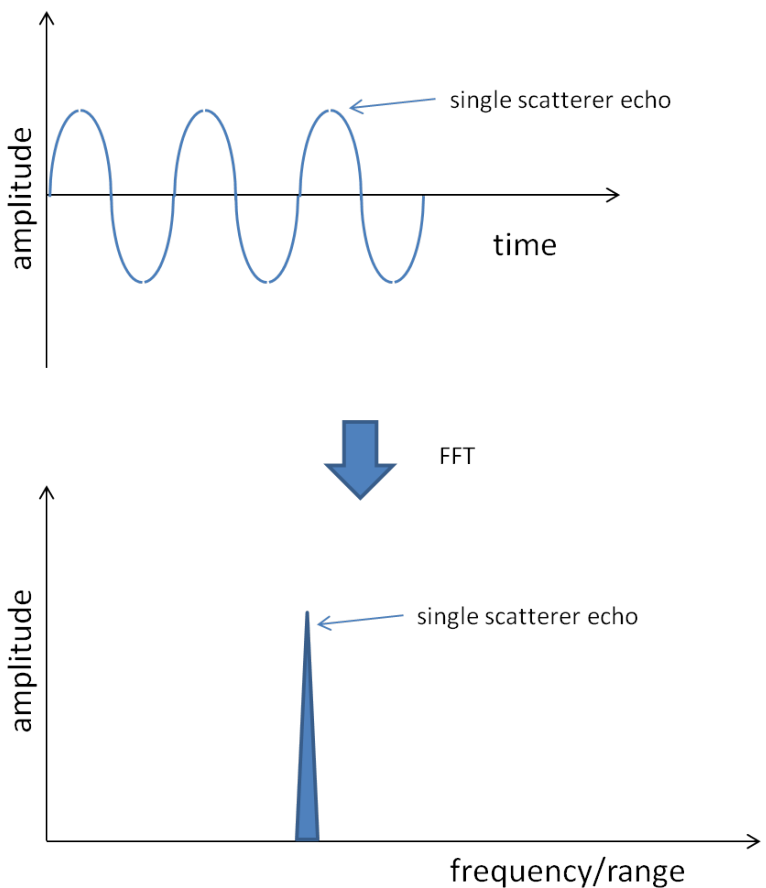

Fig. 3. The idea of FMCW range compression.

The shift of the echo may not coincide with the size of the range resolution cell, therefore fractional shift is required. This can be easily achieved by using the well known property of the Fourier transform - the shift in time domain corresponds to multiplication of the spectrum by an exponential function with linear phase. Using this property, arbitrary time shift can be obtained by multiplying the spectrum of the signal by appropriate function.

In general, the shape of the migration of the target echo has a nonlinear form. However, for relatively short observation times the nonlinear shape can be approximated by a secondorder polynomial. In such a case the range cell migration correction can be compensated according to:

$$
X_{m}(t)=X(t) \exp \left(2 \pi j\left(t c_{1}+t^{2} c_{2}\right)\right)
$$

where $X(t)$ is the signal spectrum from a single pulse (or sweep in FMCW radar), $t$ is a slow time, and $c_{1}$ and $c_{2}$ are the coefficients used for approximation of the nonlinear migration effect.

The concept of range cell migration correction is illustrated in Fig. 4. The target echo is spread across different range cells. After the range cell migration correction the echo should be within one range resolution cell.

\section{Doppler Centroid Compensation}

After the range cell migration correction the target spectrum may not be centered around zero frequency. This is due the geometry of the scene - if the target motion is not perpendicular to the line of sight, the mean Doppler frequency (the so-called Doppler centroid) will be different from zero. To compensate for this effect, the mean frequency of the signal spectrum is estimated [6], and shift to the spectrum is 

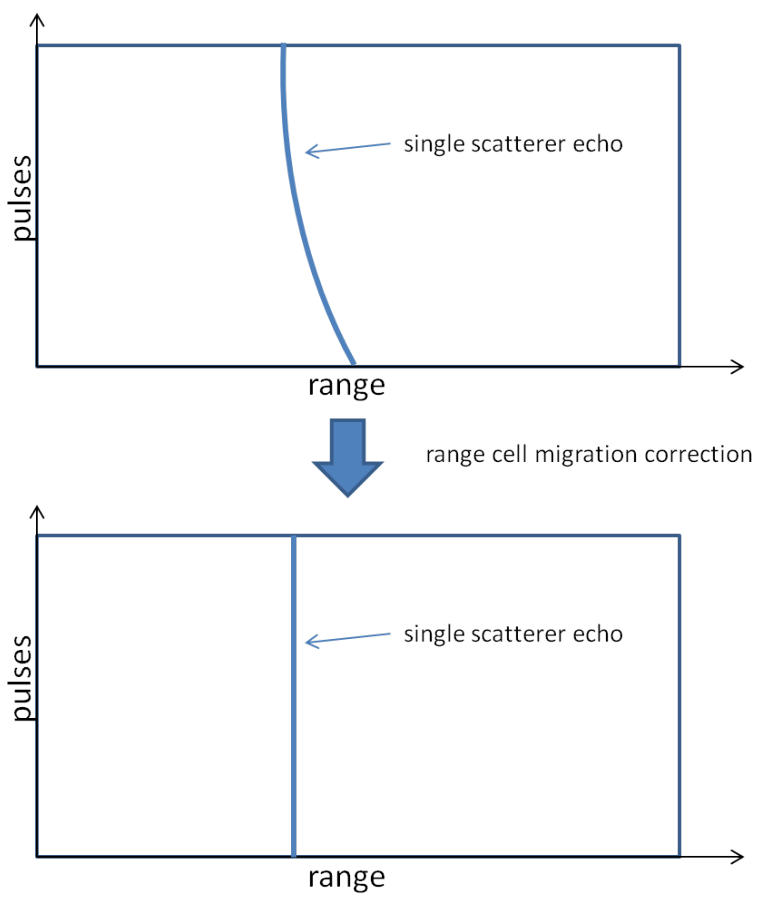

Fig. 4. The idea of range cell migration correction.

introduced. The idea of Doppler centroid correction is shown in Fig. 5.

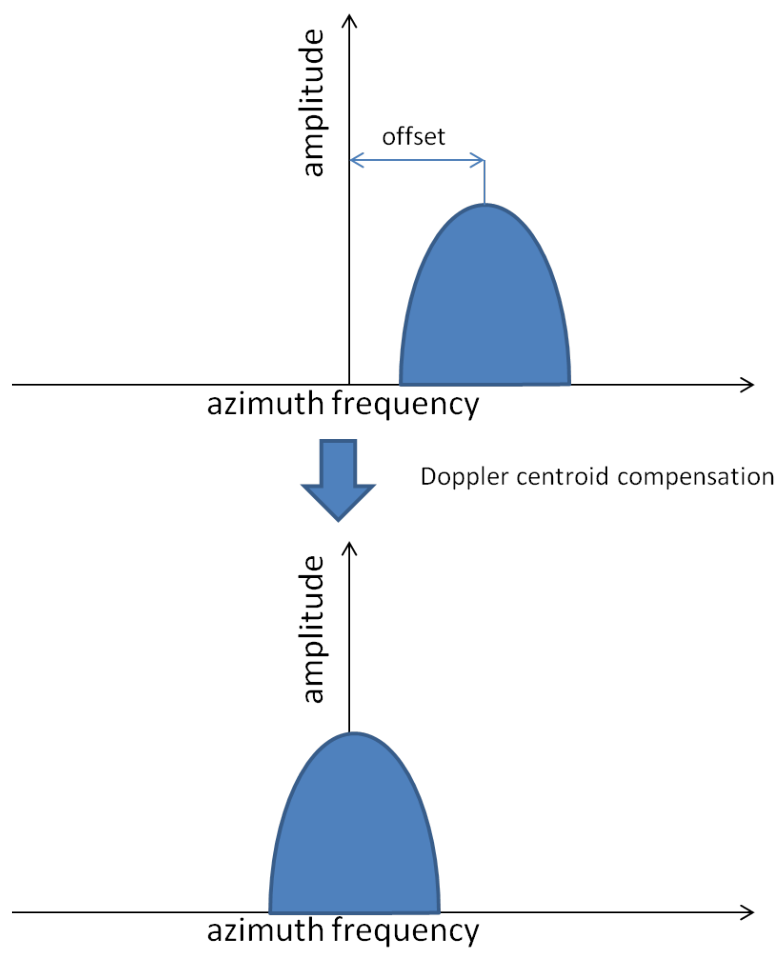

Fig. 5. The idea of Doppler centroid correction.

1) Unfocused ISAR imaging: After the aforementioned processing steps, the final imaging algorithm can be applied. As mentioned before, algorithms typical for SAR processing are used here. One of the SAR processing modes is the so- called unfocused SAR mode. In this case the signal from each range cell is filtered using a simple low-pass filter. As the signal received from a single scatterer can be approximated by a chirp signal (signal with linear frequency modulation), low-pass filtering of such signal causes a peak in the output signal to appear when the frequency of the input signal is close to 0 . In such a way, azimuth compression is obtained [6]-[9].

The unfocused SAR processing is suboptimal, and does not provide maximum obtainable resolution. However, this simple technique has moderate computational requirements, and it is robust to the mismatch of the assumed and actual target motion parameters.

2) Focused ISAR imaging: The focused SAR processing consists of matched filtering of the signal from each range cell. The matched filtering provides the maximum obtainable resolution, however, it is computationally requiring and is highly sensitive to incorrect algorithm parameters. The last disadvantage is often cumbersome in practice, i.e. the matched filter has to be designed with very high accuracy, which requires knowledge of the motion parameters of the target. In this paper this problem is solved as follows. The filter is calculated by matching the quadratic phase to the signal phase corresponding to the range cell with maximum power. This approach can be justified in the following way. Usually the observed targets are composed of a relatively small number of dominant scatterers. Very often only a single dominant scatterer is present in a single resolution cell. Such a signal (signal of a single scatterer from one resolution cell) can be used to construct nearly perfect matched filter - taking into account the real motion of the target.

The comparison of unfocused SAR processing and focused SAR processing approaches are shown in Fig. 6. Frequency of the signal originating from a single scatterer can be approximated by a chirp signal. In unfocused SAR a low-pass filter is used (marked with horizontal dashed lines), whereas in focused SAR a matched filter tuned to the received signal is applied (marked with dashed lines parallel to the signal frequency).

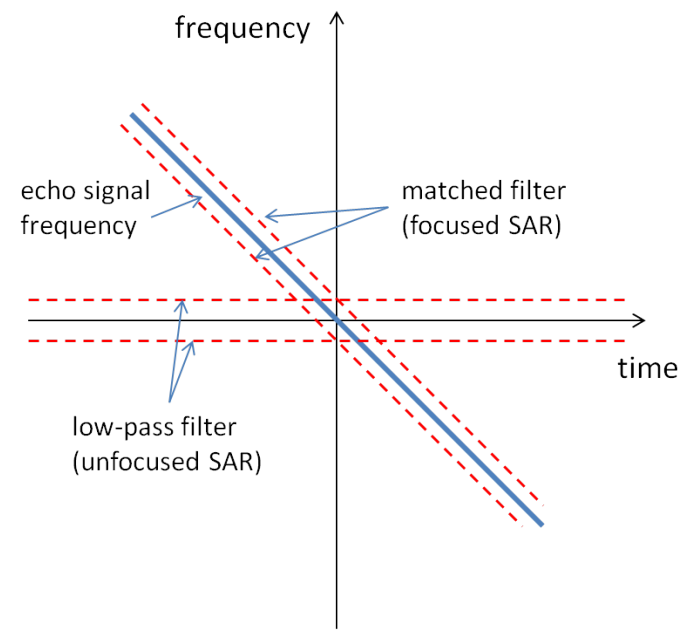

Fig. 6. Comparison of unfocused SAR and focused SAR processing approaches. 


\section{Simulations}

As a first step of the experiment, a simulation of a single ground moving target has been performed. The parameters of the simulation corresponded roughly to those encountered during measurement campaign. The target is represented as a set of four scattering points placed at its corners. The dimensions of target correspond to dimensions of an average truck. The sketch of simulated scene is presented in Fig. 7. In the simulation it was assumed that the truck is moving linearly and uniformly at a speed $v$ of $70 \mathrm{~km} / \mathrm{h}$ at an angle $\gamma$ of 60 degrees to the line of sight. The initial distance to the target $R$ is set to $550 \mathrm{~m}$ with $y_{0}$ set to $0 \mathrm{~m}$.

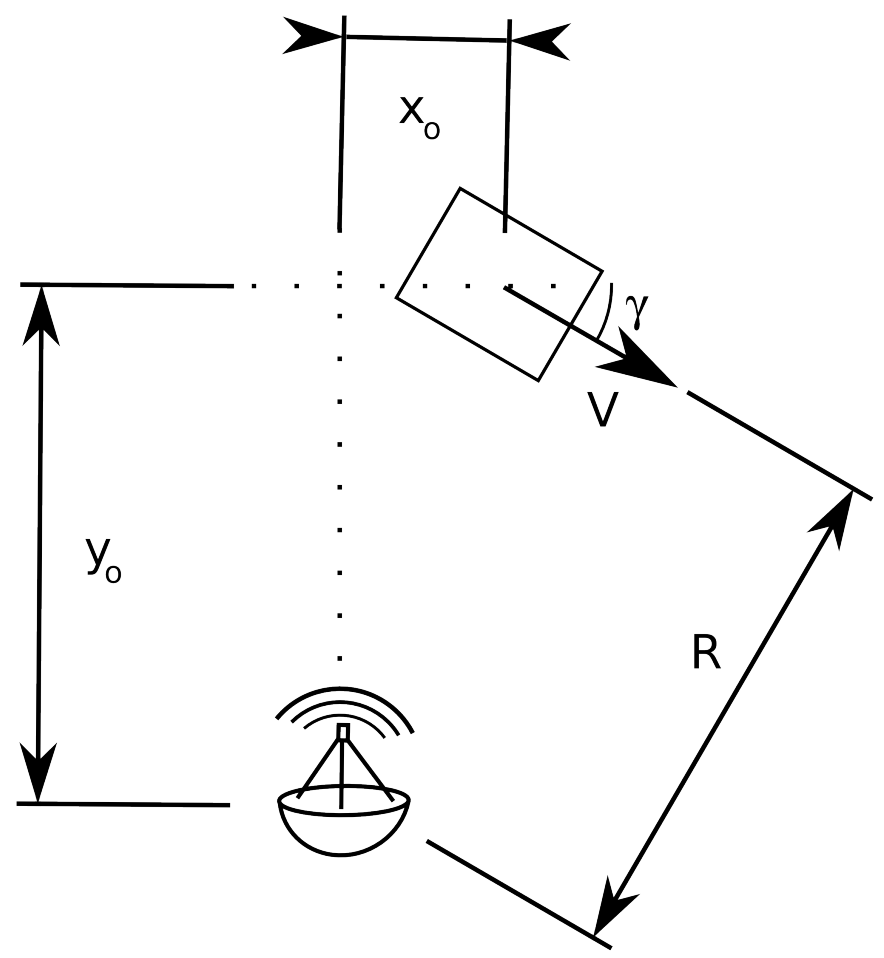

Fig. 7. The simulation scene setup.

The simulation of the radar signal has been carried out using SarSim Simulator [10], [11]. SarSim is a raw radar data simulator, which is based on the geometrical optics approach and electromagnetic approach. In presented simulation only geometrical optics approach was used. Simulation time was $1 \mathrm{~s}$ with sweep repetition frequency equal to $2000 \mathrm{~Hz}$, which results in 2000 sweeps. Carrier frequency $f_{c}=94 \mathrm{GHz}$ and bandwidth of signal $B=1 \mathrm{GHz}$.

\section{A. Range Compression}

The results after the range compression for the simulated scenario are shown in Fig. 8. In each sweep the range to target differs due to its movement. This effect is visible in the curvature of scattering points responses.

\section{B. Range Cell Migration Correction}

The second step in the algorithm is movement compensation. The signal in each pulse is shifted according to (1). The

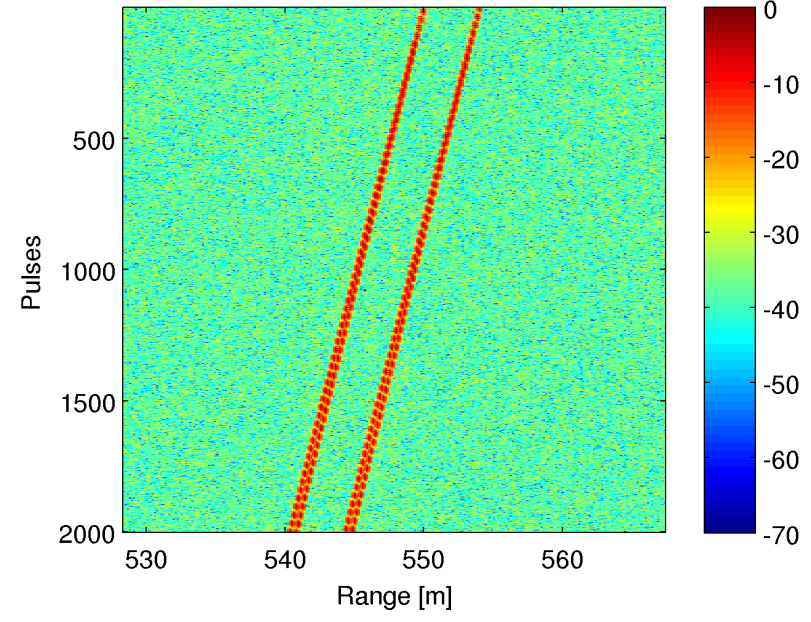

Fig. 8. Data after range compression.

result of this step is presented in Fig. 9. Neither the linear nor quadratic phase are exactly compensated but after this step the point response lies within single resolution cell.

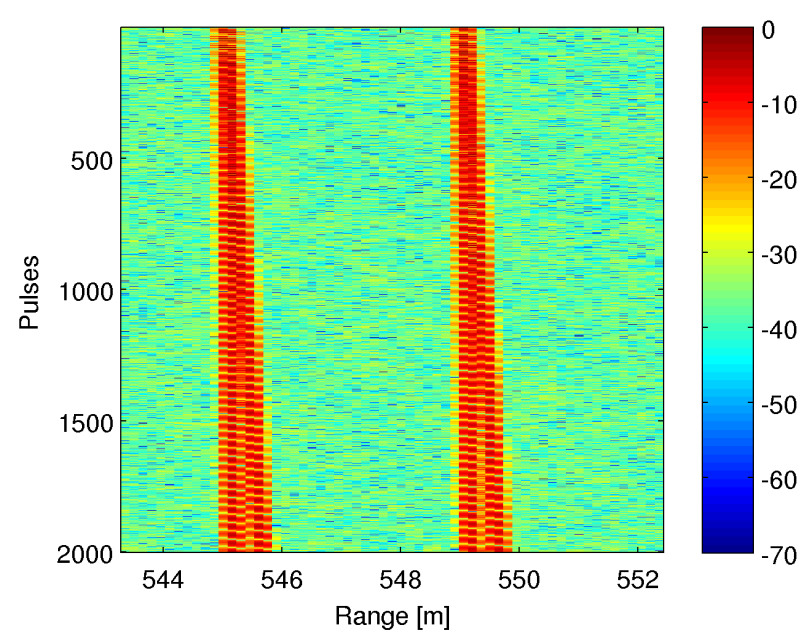

Fig. 9. Data obtained after movement compensation.

\section{Doppler Centroid Compensation}

The third step is the elimination of spectrum shift. To do this, the target spectrum shift is calculated and eliminated by signal modulation.

\section{Unfocused ISAR Imaging}

Due to the long observation time and translational motion of the target, the frequency of the signal changes approximately linearly with time, similar as in stripmap SAR. As a reference, the unfocused SAR processing has been applied to the data. The result for simulated data is presented in Fig. 10. The focusing effect can be seen - four simulated scatterers are visible. However, the resolution of the obtained image is not very satisfactory. 


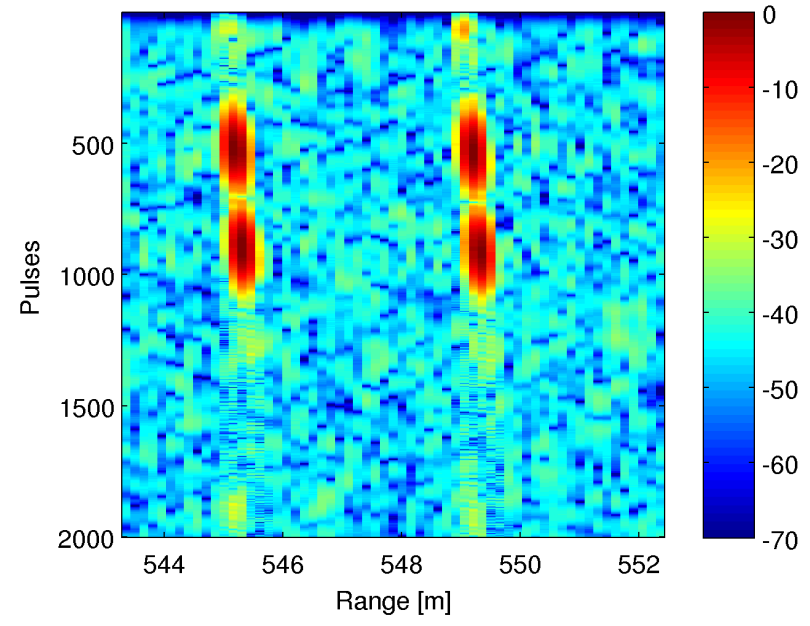

Fig. 10. Image obtained using unfocused SAR processing.

\section{E. Focused ISAR Imaging}

The focused SAR processing consists of matched filtering of the signal from each range cell. The filter is calculated by matching the quadratic phase to the phase of range cell with maximum power. Afterwards, the azimuth compression is done for each range cell by filtering the data with the designed filter. Figure 11 shows the final image of simulated data. In this case, the obtained resolution is much better than in the case of unfocused SAR technique.

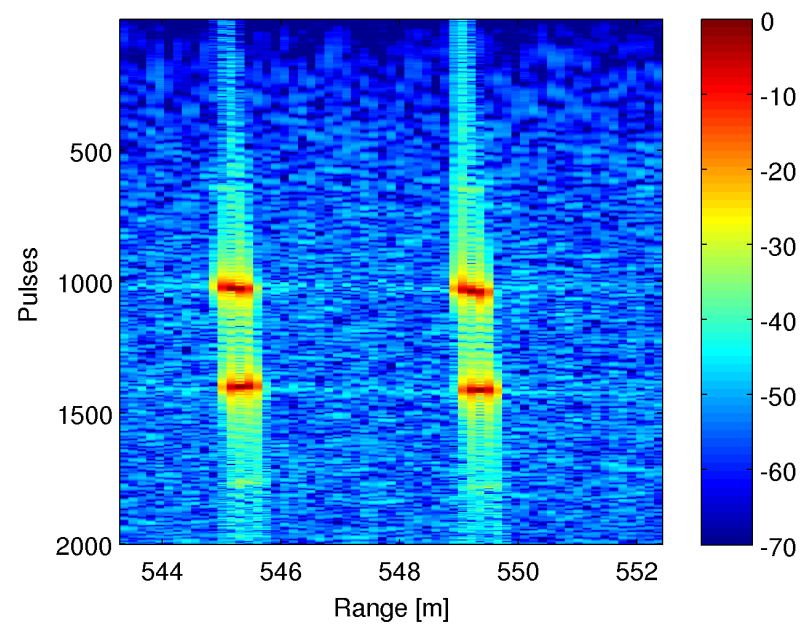

Fig. 11. Image obtained using focused SAR processing.

\section{FIELd TRIALS}

The real-data trials were performed from a window of laboratory with radar looking at a country road. The road was angled at about 30 degrees to line of sight. In experiment several non-cooperative targets were recorded including a bus, a car (Fig. 12) and a tractor (Fig. 13).

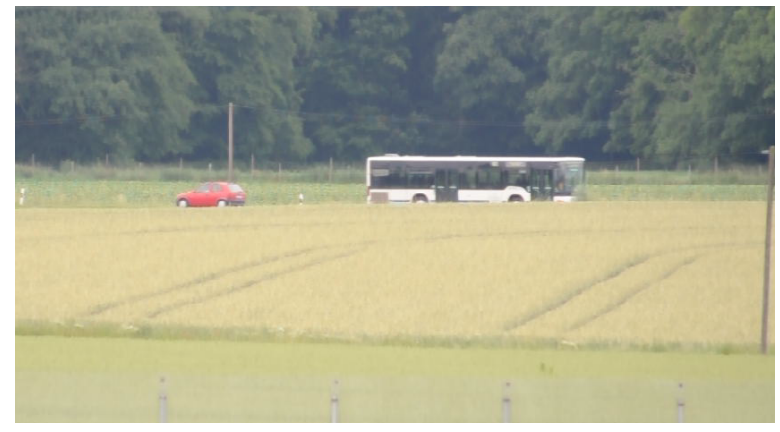

Fig. 12. A bus and a car - non-cooperative targets.

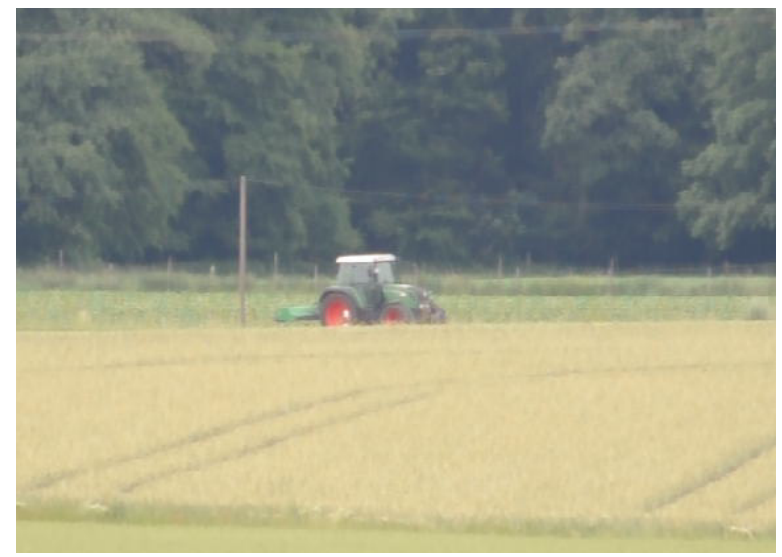

Fig. 13. A tractor - non-cooperative target.

\section{A. System Setup}

The radar used in experiment was operating at carrier frequency $f_{c}=94 \mathrm{GHz}$. The FMCW signal bandwidth $B$ was equal to $960 \mathrm{MHz}$ with chirp repetition frequency $1818 \mathrm{~Hz}$, which nearly corresponds to simulation setup.

An example of the range profiles of the observed target (a bus) after range cell migration correction is shown in Fig. 14. As can be seen the range cell migration has been corrected, however, amplitude of the scatterers varies strongly with time.

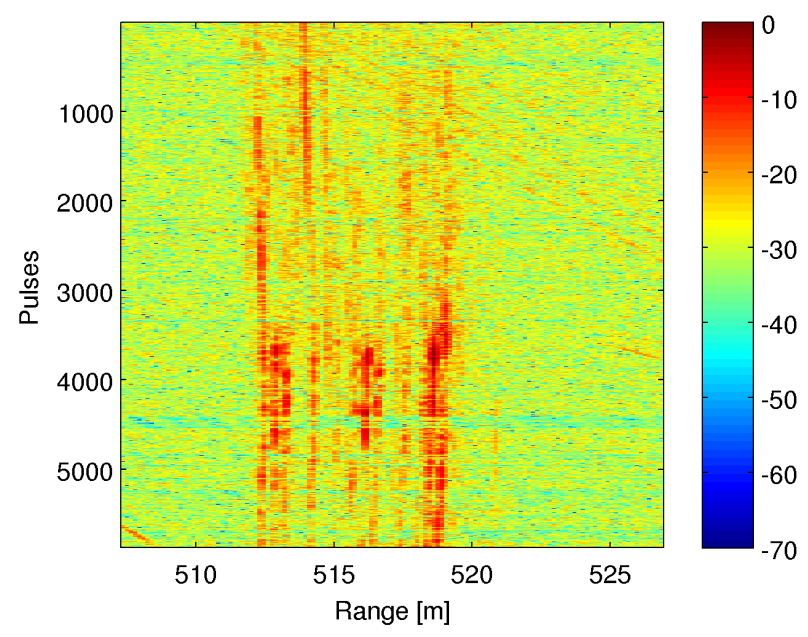

Fig. 14. Range profiles of a bus after motion compensation. 
An example of the ISAR image obtained by applying unfocused SAR processing is shown in Fig. 15. The shape of the target (the bus from the previous example) can be easily distinguishable. However, as mentioned before, the unfocused SAR processing is suboptimal in terms of the obtained resolution. The result obtained by applying the focused SAR processing to the same data set is shown in Fig. 16. An increase in azimuth resolution is clearly seen.

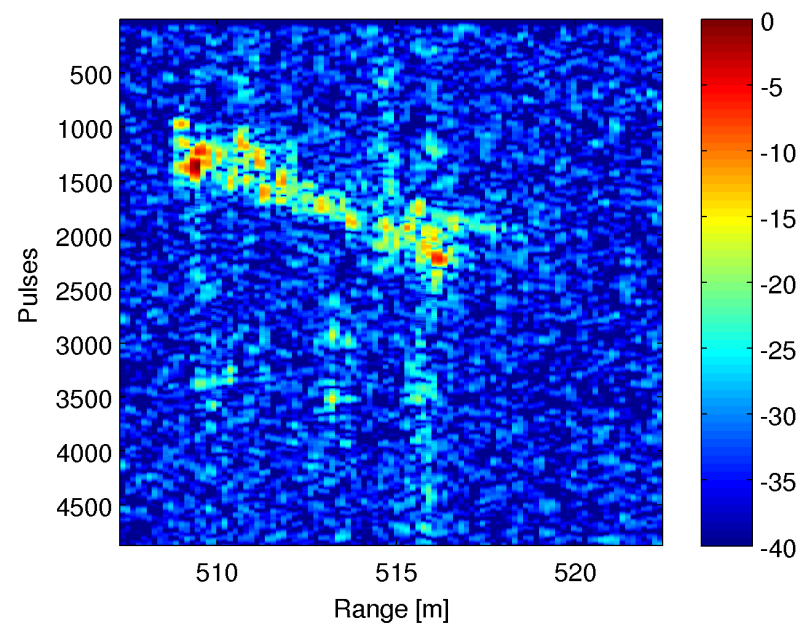

Fig. 15. ISAR image of a bus (unfocused SAR processing).

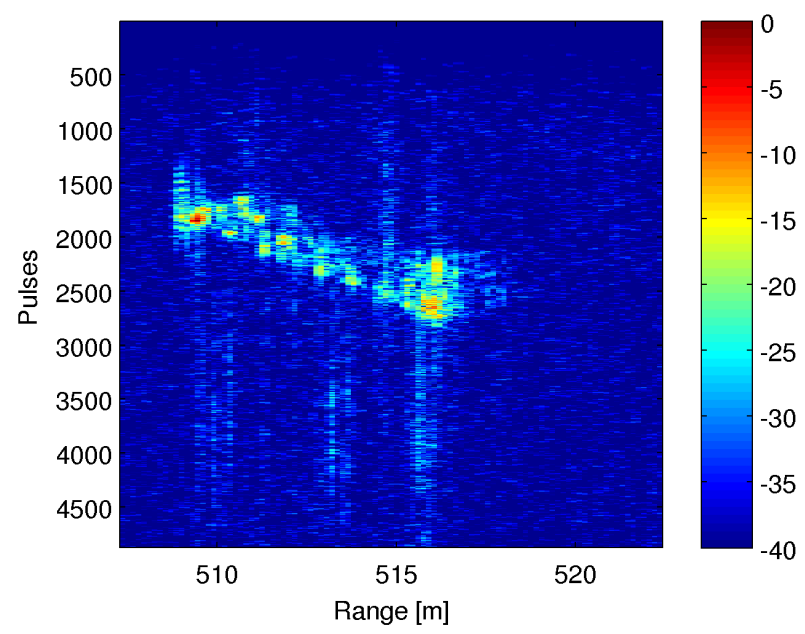

Fig. 16. ISAR image of a bus (focused SAR processing).

Other examples of ISAR imaging (using the focused SAR processing) of targets are shown in Figs. 17 and 18. The full shape of the target is not always visible, due to the shadowing effect - the radar illuminates only one side of the target. However, general characteristics of the target, such as range extent, can be inferred from the obtained images.

\section{CONCLUSION}

The high resolution ISAR imaging is technique that allows ground moving target observation. In the paper a theoretical background was presented, together with simulation and

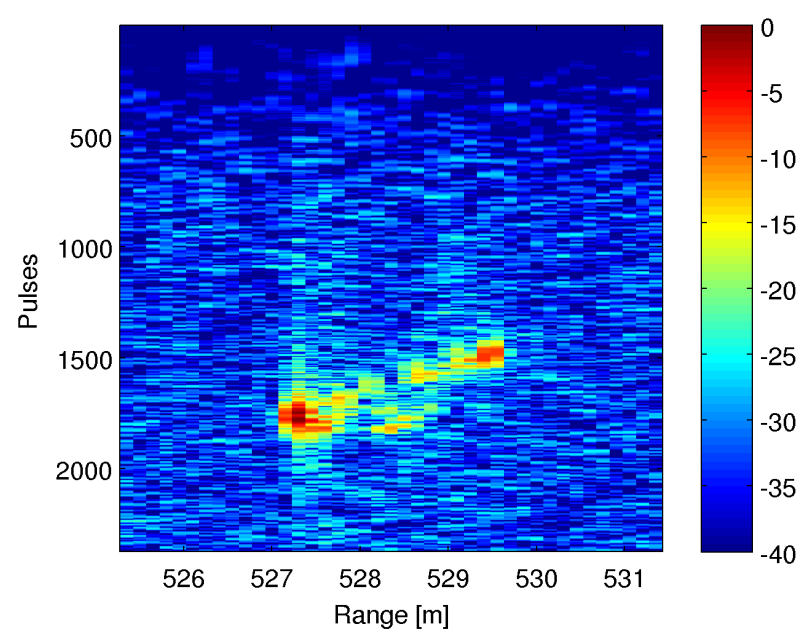

Fig. 17. ISAR image of a car.

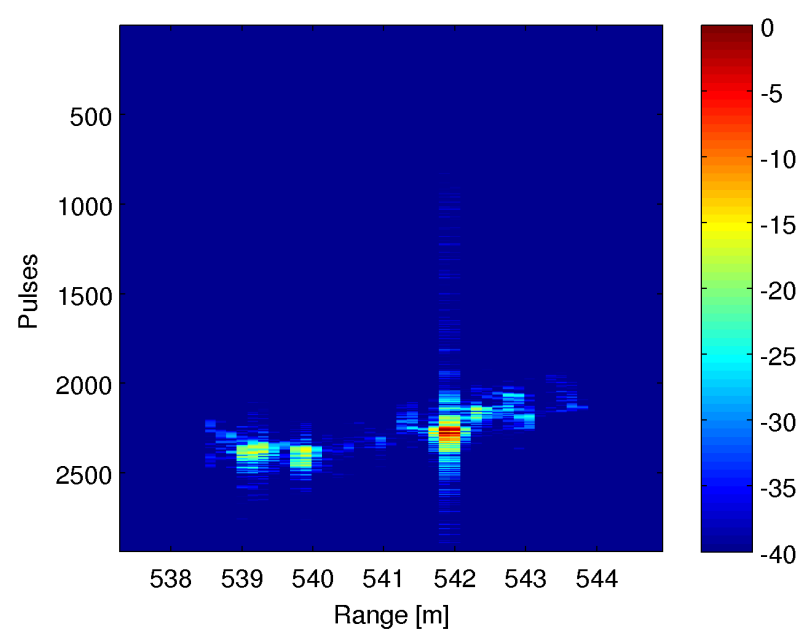

Fig. 18. ISAR image of a tractor.

measurement results. Due to the unusual scenario of ISAR imaging, i.e. long observation time and high lateral velocity component, algorithms known from SAR were applied. As a result, high-resolution images have been obtained.

The obtained images allow to roughly determine the size of the targets, and identify their characteristic features. This can be a basis for classification and recognition. This feature would be useful in traffic monitoring, as not only number of vehicles could be determined, but also their size or type.

\section{ACKNOWLEDGMENT}

The authors would like to thank Michael Caris, Stephan Stanko, Rainer Sommer and Winfried Johannes from Fraunhofer FHR and Arnulf Leuther from Fraunhofer IAF for providing the analog frontend and organizing the measurement campaign. The authors would also like to thank Michael Cohen and Peter Kovacs form ProPatria Electronics for supporting in the measurement campaign and preparation of analog-todigital conversion unit. 


\section{REFERENCES}

[1] D. R. Wehner, High-Resolution Radar, 2nd ed. Artech House, Inc., Dec. 1994

[2] J. S. Kulpa, D. Gromek, A. Gromek, P. Samczyski, M. Malanowski, and K. Kulpa, "Results of high-resolution isar imaging of ground moving targets," in Proc. Signal Processing Symposium 2013, 2013, p. CD.

[3] M. Caris, S. Stanko, H. Essen, A. Leuther, A. Tessmann, R. Weber, M. Malanowski, P. Samczynski, K. Kulpa, G. Meszoly, A. Papanastasiou, C. Topping, G. Georgiou, R. Guraly, and Z. Bilacz, "Synthetic aperture radar for all weather penetrating UAV application (SARAPE) - project presentation," in Proc. EUSAR, 2012, pp. 290-293.

[4] M. Caris, S. Stanko, R.Sommer, A. Wahlen, A. Leuther, A. Tessmann, M. Malanowski, P. Samczyski, K. Kulpa, M. Cohen, P. Kovacs, A. Papanastasiou, C. Topping, G. Georgiou, and R. Guraly, "SARape - synthetic aperture radar for all weather penetrating UAV application,' in Proc. International Radar Symposium IRS 2013, 2013.

[5] M. Malanowski, G. Krawczyk, P. Samczyski, K. Kulpa, K. Borowiec, and D. Gromek, "Real-time high-resolution SAR processor using CUDA technology," in Proc. International Radar Symposium IRS 2013, 2013.
[6] K. Kulpa, M. Purchla-Malanowska, and M. P. Malanowski, "Real-time unfocused synthetic aperture radar algorithm with motion compensation," in Proc. International Radar Symposium IRS 2003, 2003, pp. 6772.

[7] M. Purchla-Malanowska and M. P. Malanowski, "Simple motion compensation algorithm for unfocused synthetic aperture radar," in XII IEEESPIE Symposium on Photonics and Web Engineering, 2003, pp. CDROM proceedings.

[8] _ - "Simple motion compensation algorithm for unfocused synthetic aperture radar," in Proceedings of SPIE: Photonics Applications in Astronomy, Communications, Research and High Energy Physics Experiments II, 2004, pp. 659-664.

[9] K. Kulpa, M. Purchla-Malanowska, and M. P. Malanowski, "Improvement of resolution in real-time unfocused sar algorithm," in Proc. EUSAR 2004. 5th European Conference on Synthetic Aperture Radar, 2004, pp. 725-728.

[10] G. Tanski, "Software radar echo synthesizer," Proc. of SPIE, vol. 5484, pp. 696-701, 2004.

[11] D. Gromek, A. Gromek, K. Kulpa, M. Malanowski, P. Samczyski, and G. Taski, "SAR/InSAR raw data simulator using 3D terrain model," Elektronika-konstrukcje, technologie, zastosowania, pp. 44-46, 2004. 